\title{
Apoptosis Induced by Tanshinone IIA and Cryptotanshinone Is Mediated by Distinct JAK/STAT3/5 and SHP1/2 Signaling in Chronic Myeloid Leukemia K562 Cells
}

\author{
Ji Hoon Jung, ${ }^{1}$ Tae-Rin Kwon, ${ }^{1}$ Soo-Jin Jeong, ${ }^{1,2}$ Eun-Ok Kim, ${ }^{1}$ \\ Eun Jung Sohn, ${ }^{1}$ Miyong Yun, ${ }^{1}$ and Sung-Hoon Kim ${ }^{1}$ \\ ${ }^{1}$ College of Oriental Medicine, Kyung Hee University, 1 Hoegi-dong, Dongdaemun-gu, Seoul 130-701, Republic of Korea \\ ${ }^{2}$ Basic Herbal Medicine Research Group, Herbal Medicine Research Division, Korea Institute of Oriental Medicine, \\ Daejeon 305-811, Republic of Korea \\ Correspondence should be addressed to Sung-Hoon Kim; sungkim7@khu.ac.kr
}

Received 4 April 2013; Accepted 3 June 2013

Academic Editor: Khalid Rahman

Copyright (C) 2013 Ji Hoon Jung et al. This is an open access article distributed under the Creative Commons Attribution License, which permits unrestricted use, distribution, and reproduction in any medium, provided the original work is properly cited.

\begin{abstract}
Though tanshinone IIA and cryptotanshinone possess a variety of biological effects such as anti-inflammatory, antioxidative, antimetabolic, and anticancer effects, the precise molecular targets or pathways responsible for anticancer activities of tanshinone IIA and cryptotanshinone in chronic myeloid leukemia (CML) still remain unclear. In the present study, we investigated the effect of tanshinone IIA and cryptotanshinone on the Janus activated kinase (JAK)/signal transducer and activator of transcription (STAT) signaling during apoptotic process. We found that both tanshinone IIA and cryptotanshinone induced apoptosis by activation of caspase-9/3 and Sub-G1 accumulation in K562 cells. However, they have the distinct JAK/STAT pathway, in which tanshinone IIA inhibits JAK2/STAT5 signaling, whereas cryptotanshinone targets the JAK2/STAT3. In addition, tanshinone IIA enhanced the expression of both SHP-1 and -2, while cryptotanshinone regulated the expression of only SHP-1. Both tanshinone IIA and cryptotanshinone attenuated the expression of $b_{c l} x_{\mathrm{L}}$, survivin, and cyclin D1. Furthermore, tanshinone IIA augmented synergy with imatinib, a CML chemotherapeutic drug, better than cryptotanshinone in K562 cells. Overall, our findings suggest that the anticancer activity of tanshinone IIA and cryptotanshinone is mediated by the distinct the JAK/STAT3/5 and SHP1/2 signaling, and tanshinone IIA has the potential for combination therapy with imatinib in K562 CML cells.
\end{abstract}

\section{Introduction}

Salvia miltiorrhiza Bunge (Danshen) is a traditional medicinal herb widely used for treating cardiovascular disease in Korea, China, and Japan [1]. To date, over 90 kinds of chemical constituents from $S$. miltiorrhiza have been reported $[2,3]$. Of the phytochemicals, tanshinones are a group of lipophilic abietane diterpene compounds including tanshinone I, tanshinone IIA-B, cryptotanshinone, dihydrotanshinone I, isotanshinone I, and isocryptotanshinone I-II and have been extensively investigated [1, 4-7]. In particular, tanshinone IIA and cryptotanshinone have been presented the potential as anticancer drugs by targeting the multiple signaling pathways [8-18].
STAT family is transcriptional factors that play key roles in cytokine signaling [19]. STAT proteins are constitutively activated in cancer cells or tissues and thus have been suggested as attractive molecular target(s) for cancer therapy. In light of these events, numerous groups reported the inhibitory effects of plant polyphenols such as curcumin, resveratrol, piceatannol, and EGCG on STAT activation in various cancer cells $[19,20]$. Tanshinone IIA and cryptotanshinone were also shown to have the inhibitory effects on the STAT activation in C6 glioma [21] and DU145 prostate cancer cells [22], respectively. However, there is no report on the molecular mechanisms leading to anticancer activity of tanshinone IIA and cryptotanshinone through the STAT signaling pathway in leukemia cells. 
In the current study, we investigated the inhibitory effects of tanshinone IIA and cryptotanshinone on the activation of STAT3 or 5 linked to apoptosis in chronic myeloid leukemia (CML) K562 cells. Additionally, the synergistic effects of tanshinone IIA or cryptotanshinone with imatinib, a chemotherapeutic agent for CML, were examined by calculating combination index $(\mathrm{CI})$.

\section{Materials and Methods}

2.1. Isolation of Tanshinone IIA and Cryptotanshinone. Tanshinone IIA [23] and cryptotanshinone [24] (Figure 1(a)) were isolated as previously described.

2.2. Cell Culture. Human chronic myeloid leukemia K562 cells were purchased from American Type Culture Collection (ATCC, Rockville, MD, USA) and maintained in RPMI 1640 medium supplemented 10\% fetal bovine serum (FBS), $2 \mu \mathrm{M}$ L-glutamine, and penicillin/streptomycin.

2.3. Cytotoxicity Assay. Cytotoxic effects of tanshinone IIA or cryptotanshinone against K562 cells were evaluated by 3 (4,5-dimethylthiazol-2-yl)-2,5-diphenyltetrazolium bromide (MTT) assay. Cells were seeded onto 96-well microplates at a density of $2 \times 10^{4}$ cells per well and exposed to various concentrations of tanshinone IIA or cryptotanshinone $(0,10$, $20,40$, or $80 \mu \mathrm{M})$ for $24 \mathrm{~h}$. The cells were incubated with 3(4,5-dimethylthiazol-2-yl)-2,5-diphenyl tetrazolium bromide ( $1 \mathrm{mg} / \mathrm{mL}$ ) (Sigma Chemical Co., St. Louis, MO, USA) for $2 \mathrm{~h}$ and then with MTT lysis solution overnight. Optical density (OD) was measured using a microplate reader (Molecular Devices Co., Sunnyvale, CA, USA) at $570 \mathrm{~nm}$. Cell viability was calculated as a percentage of viable cells in drug-treated group versus untreated control by the following equation:

$$
\begin{aligned}
& \text { Cell viability }(\%) \\
& \qquad=\frac{[\mathrm{OD}(\text { Drug })-\mathrm{OD}(\text { Blank })]}{[\mathrm{OD}(\text { Control })-\mathrm{OD}(\text { Blank })]} \times 100 .
\end{aligned}
$$

2.4. Western Blot Analysis. K562 cells were lysed in lysis buffer $(50 \mathrm{mM}$ Tris-HCl, pH 7.4, $150 \mathrm{mM} \mathrm{NaCl}, 1 \%$ Triton X100, 0.1\% SDS, $1 \mathrm{mM}$ EDTA, $1 \mathrm{mM} \mathrm{Na} \mathrm{VO}_{4}, 1 \mathrm{mM} \mathrm{NaF}$, and protease inhibitors cocktail). The extracts were incubated on ice for $30 \mathrm{~min}$ and supernatants were collected by centrifugation at $14,000 \mathrm{~g}$ at $4^{\circ} \mathrm{C}$. The protein contents in the supernatant were measured by using a Bio-Rad DC protein assay kit II. Proteins were separated by electrophoresis on $12.5 \%$ SDSPAGE gel and electrotransferred onto a Hybond ECL transfer membrane with transfer buffer (25 mM Tris, $250 \mathrm{mM}$ glycine, and $20 \%$ methanol) at $300 \mathrm{~mA}$ for $90 \mathrm{~min}$. The membrane was blocked in 5\% nonfat skim milk and probed with primary antibodies for p-STAT3, p-STAT5, STAT3, STAT5, p-JAK2, JAK2 (Cell Signaling Tech., Danvers, MA, USA), SHP-1, SHP-2, bcl- $\mathrm{x}_{\mathrm{L}}$, mcl-1, survivin, cyclin D1, cleaved caspase9, cleaved caspase-3, poly(ADP-ribose) polymerase (PARP), and tubulin (Santa Cruz Biotechnologies, Santa Cruz, CA, USA), followed by incubating with horseradish peroxidase(HRP-) conjugated secondary antibodies. Protein expression was detected by using enhanced chemiluminescence (ECL) system (Amersham Pharmacia, Piscataway, NJ, USA).

2.5. Electrophoretic Mobility Shift Assay (EMSA). The STAT3 or STAT5/DNA binding activity was analyzed by EMSA using gel shift chemiluminescent EMSA kit (Active motif, Carlsbad, CA, USA). Nuclear extracts were incubated with STAT3 (5'-GAT CCT TCT GGG AAT TCC TAG ATC-3') or STAT5 (5'-AGA TTT CTA ATT CAA TCC- $\left.3^{\prime}\right)$ consensus oligonucleotides (Santa Cruz Biotechnology, Santa Cruz, CA, USA). The DNA/protein complex formed was separated from free oligonucleotides on $5 \%$ native polyacrylamide gels. Chemiluminescent detection was performed using ECL reagents according to the vendor's protocols (GE Health Care Bio-Sciences, Piscataway, NJ, USA).

2.6. Cell Cycle Analysis. Cell cycle analysis was performed by PI staining. K562 cells were treated with tanshinone IIA or cryptotanshinone for $24 \mathrm{~h}$, collected and fixed in $70 \%$ ethanol. The cells were then incubated at $37^{\circ} \mathrm{C}$ with $0.1 \%$ RNase A in PBS for $30 \mathrm{~min}$ and suspended in PBS containing $25 \mu \mathrm{g} / \mathrm{mL}$ PI for $30 \mathrm{~min}$ at room temperature. The stained cells were analyzed for DNA content in FACSCalibur (Becton Dickinson, Franklin Lakes, NJ, USA) using the Cell Quest program (Becton Dickinson, Franklin Lakes, NJ, USA).

2.7. Apoptosis Analysis by Annexin V-PI Double Staining. Apoptosis of the cryptotanshinone or tanshinone IIA-treated cells was quantitated by double staining with Annexin VFITC and PI using the Annexin V-Apoptosis Detection kit (Biovision, Milpitas, CA, USA) according to the manufacturer's instructions. Apoptotic cells were analyzed by FACSCalibur (Becton Dickinson, San Jose, CA, USA) to be defined as those positive for Annexin V with or without PI staining.

2.8. Propidium Iodide (PI) Staining. K562 cells were exposed to tanshinone IIA or cryptotanshinone and plated onto polyL-lysine-coated slide glass. The cells were fixed in $70 \%$ ethanol and stained with PI solution $(50 \mu \mathrm{g} / \mathrm{mL}$ ) (BD Biosciences, Bedford, MA, USA) containing $100 \mu \mathrm{g} / \mathrm{mL}$ RNase for $10 \mathrm{~min}$. The slides were mounted with 70\% glycerol in PBS and visualized under an Axio vision 4.0 fluorescence microscope (Carl Zeiss Inc., Weimar, Germany).

2.9. Combination Index (CI) Calculation. The CI was determined by Chou-Talalay method and CalcuSyn software (Biosoft, Ferguson, MO, USA). A CI of less than 1 was considered synergistic [25].

2.10. Statistical Analyses. All data were presented as means \pm standard deviation (SD). Statistical significance was verified by Student's $t$-test using SigmaPlot software (Systat Software Inc., San Jose, CA, USA).

\section{Results}

3.1. Tanshinone IIA and Cryptotanshinone Exert Cytotoxicity against Chronic Myeloid Leukemia K562 Cells. To compare the cytotoxicity of tanshinone IIA and cryptotanshinone in K562 cells, MTT assay was performed. Cells were treated 

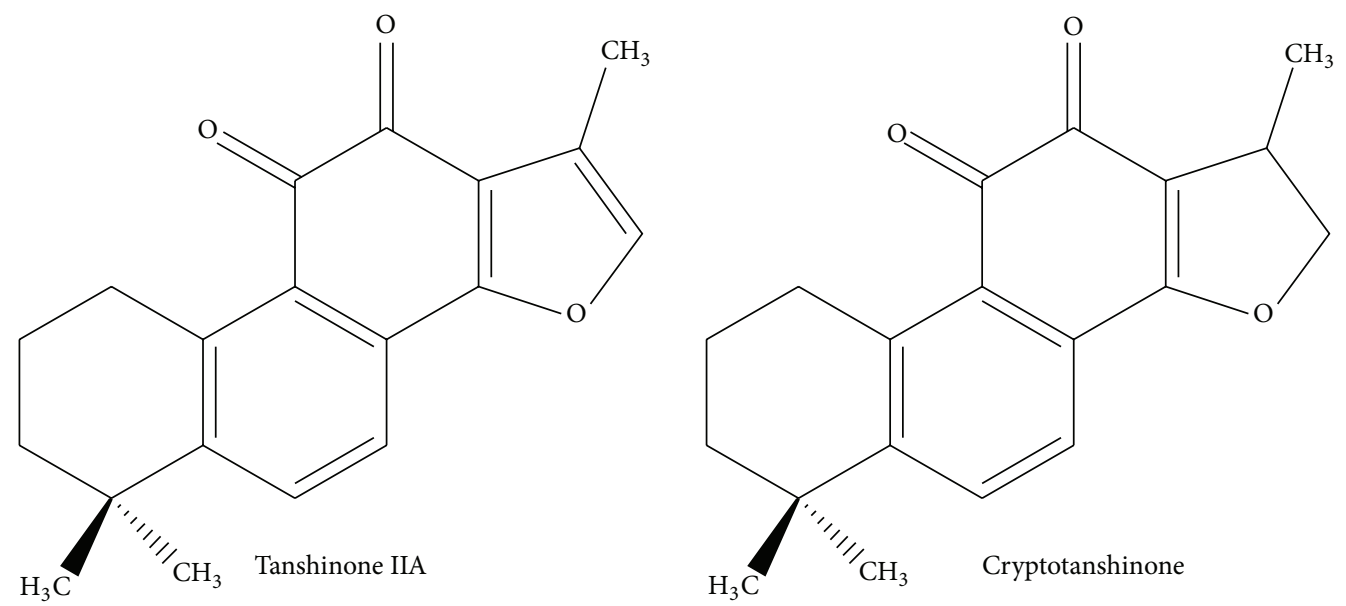

(a)

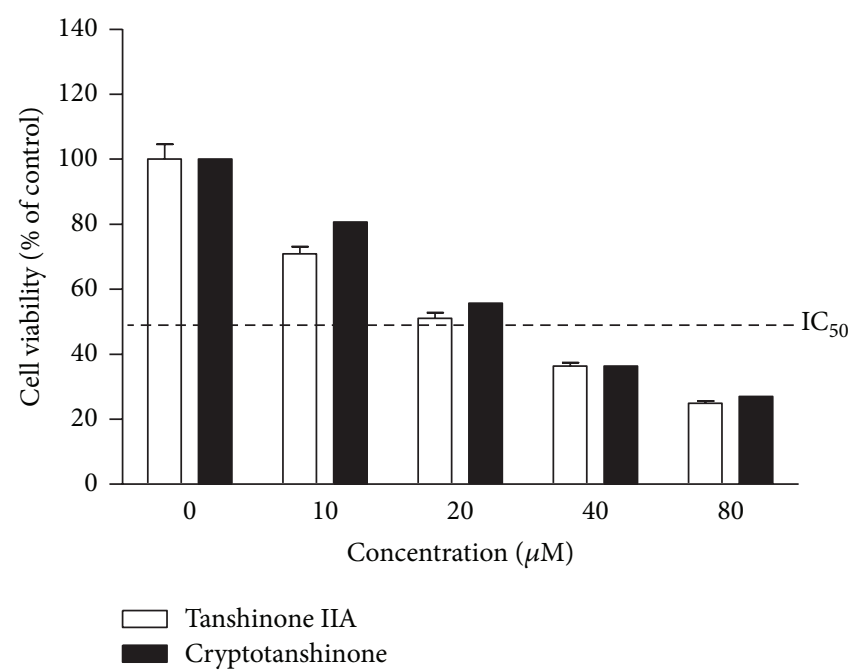

(b)

Figure 1: Tanshinone IIA and cryptotanshinone exert cytotoxicity in K562 cells. (a) Chemical structures of tanshinone IIA (left) and cryptotanshinone (right). (b) Cells were treated with various concentrations of tanshinone IIA or cryptotanshinone $(0,10,20,40$, or $80 \mu \mathrm{M})$ for $24 \mathrm{~h}$. MTT assay was performed to evaluate the cytotoxicity.

with various concentrations $(0,10,20,40$, or $80 \mu \mathrm{M})$ for $24 \mathrm{~h}$. Both tanshinone IIA and cryptotanshinone substantially reduced the cell viability in a dose-dependent manner (Figure 1(b)). There was no significant difference in the cytotoxicity between two chemicals in the cells $\left(\mathrm{IC}_{50}=\sim 20 \mu \mathrm{M}\right)$.

\subsection{Tanshinone IIA Inhibits STAT5, but Not STAT3, Signaling} in K562 Cells. Effects of tanshinone IIA on STAT3 and 5 activation were examined by Western blot analysis. As shown in Figure 2(a), tanshinone IIA treatment significantly inhibited the phosphorylation of STAT5, but not STAT3, in a dose- and time-dependent manner. We further confirmed the inhibitory effect of tanshinone IIA on STAT5 by gel shift mobility assay. Consistent with the results of immunoblotting, tanshinone IIA prevented the STAT5/DNA binding in a dose-dependent manner (Figure 2(b)). To find out whether tyrosine kinases mediate the tanshinone IIAinitiated STAT5 inactivation, the effects of tanshinone IIA on the phosphorylation of JAK1, 2 and c-Src in K562 cells were examined. The results revealed that tanshinone IIA led to dephosphorylation of JAK2 (Figure 2(c)), but not JAK1 and c-Src (data not shown). Furthermore, we observed that tanshinone IIA enhanced expression of tyrosine phosphatase SHP-1 and -2 in a time-dependent manner (Figure 2(d)).

3.3. Cryptotanshinone Inhibits STAT3, but Not STAT5, Signaling in K562 Cells. Parallel assays were carried out in cryptotanshinone-treated K562 cells. Different from tanshinone IIA, cryptotanshinone reduced the phosphorylation level of STAT3, but not STAT5, in a dose- and timedependent manner (Figure 3(a)). In addition, cryptotanshinone suppressed the binding of STAT3 to DNA in a dose-dependent manner (Figure 3(b)). However, cryptotanshinone also inhibited the phosphorylation of JAK2, an upstream kinase of STAT3 or 5 in the cells (Figure 3(c)). Besides, cryptotanshinone led to increased expression of SHP-1, but no effect on the expression of SHP-2 (Figure 3(d)). 

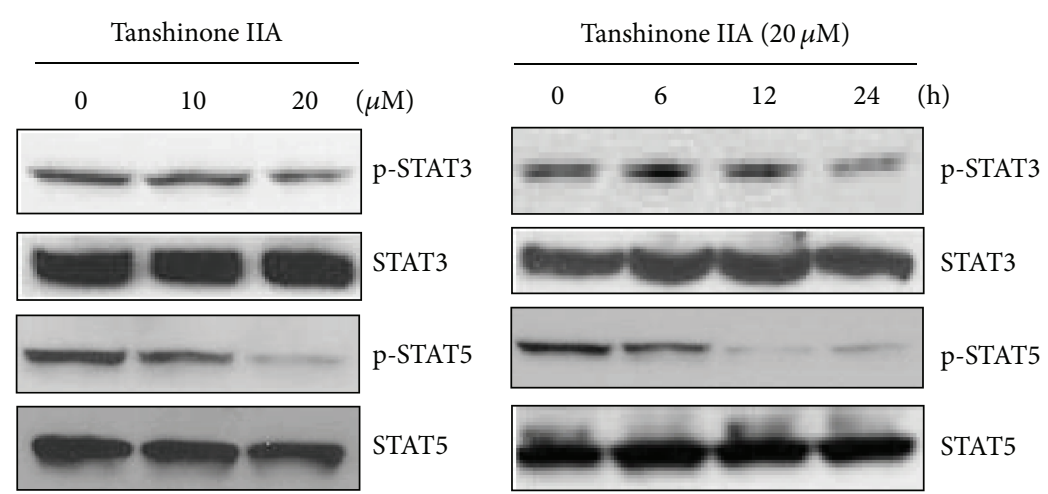

(a)

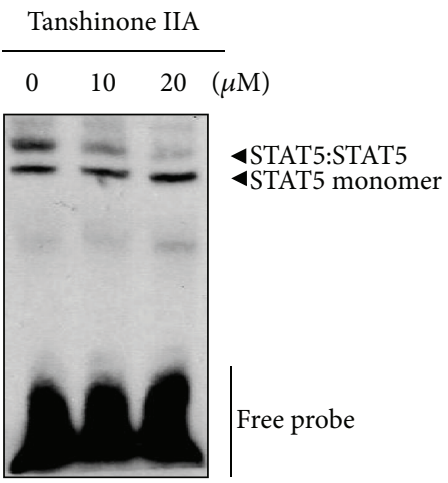

(b)

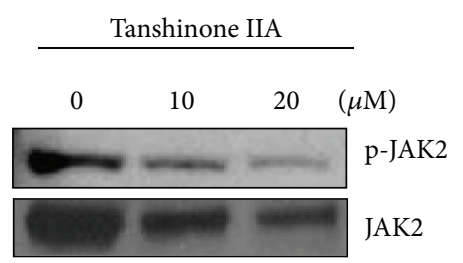

(c)

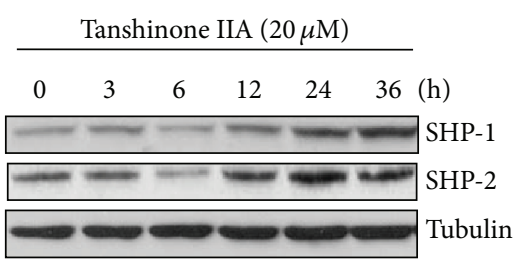

(d)

Figure 2: Tanshinone IIA inactivates STAT5, but not STAT3, in K562 cells. (a) Cells were treated with tanshinone IIA (0, 10, or 20 $\mu$ M) for $24 \mathrm{~h}$ (left) or $20 \mu \mathrm{M}$ for $0,6,12$, or $24 \mathrm{~h}$ (right). Cell lysates were prepared and subjected to Western blotting for phospho-STAT3 and phospho-STAT5. (b) Cells were treated with tanshinone IIA $(0,10$, or, $20 \mu \mathrm{M})$ for $24 \mathrm{~h}$. Gel shift mobility assay was performed to determine the STAT5/DNA binding activity. (c) Cells were treated with tanshinone IIA (0, 10, or $20 \mu \mathrm{M})$ for $24 \mathrm{~h}$. Western blotting was performed to detect phosphorylation of JAK2. (d) Cells were treated with $20 \mu \mathrm{M}$ tanshinone IIA for $0,3,6,12$, 24, or $36 \mathrm{~h}$. Western blotting was conducted to determine the expression of SHP-1 and SHP-2.

3.4. Tanshinone IIA and Cryptotanshinone Induce Apoptosis in K562 Cells. JAK/STAT signaling regulates gene products involved in various cellular processes such as survival, proliferation, and cell cycle progression [20,26, 27]. Both tanshinone IIA and cryptotanshinone significantly attenuated the expression of STAT-related survival genes such as bcl$\mathrm{x}_{\mathrm{L}}$, surviving, and cyclin D1 in a dose-dependent manner (Figure 4(a)). However, only tanshinone IIA, but not cryptotanshinone, suppressed the expression of antiapoptotic mcl$1_{\mathrm{L}}$ in K562 cells (Figure 4(a), left panel). To confirm that tanshinone IIA or cryptotanshinone can induce apoptosis, activation of caspase-9 and -3 , key molecules in intrinsic apoptosis pathway [28], was evaluated by immunoblotting. As expected, both tanshinone IIA and cryptotanshinone clearly induced the cleavages of caspase- 9 and -3 as well as
PARP in a dose-dependent manner (Figure 4(b)). Consistently, cell cycle analysis showed increased accumulation of the sub-G1 cell from $0.22 \%$ to $17.19 \%$ or $17.60 \%$ by tanshinone IIA or cryptotanshinone in K562 cells, respectively (Figure $4(\mathrm{c}))$. Moreover, we found that treatment of $20 \mu \mathrm{M}$ tanshinone IIA or cryptotanshinone dramatically increased the apoptotic cell population by Annexin V-PI double staining to 23.96 and $18.01 \%$, respectively (Figure $4(\mathrm{~d})$ ).

3.5. Tanshinone IIA and Cryptotanshinone Synergistically Promote Anticancer Effects with Imatinib in K562 Cells. Bcr$\mathrm{abl}$ is an abnormal gene formed by the reciprocal translocation between chromosomes 9 and 22 in CML [29]. We examined whether tanshinone IIA or cryptotanshinone can affect activation of bcr-abl by Western blotting. As shown 

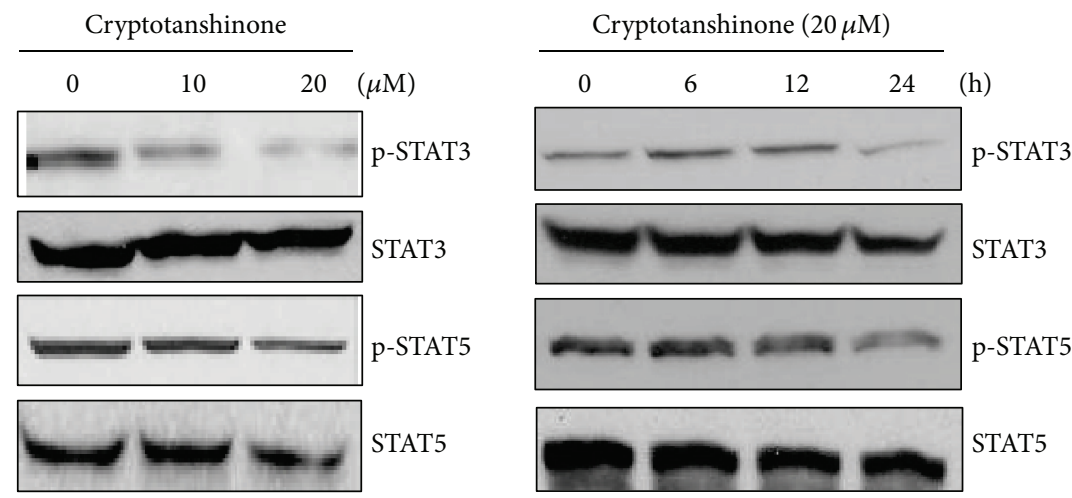

(a)

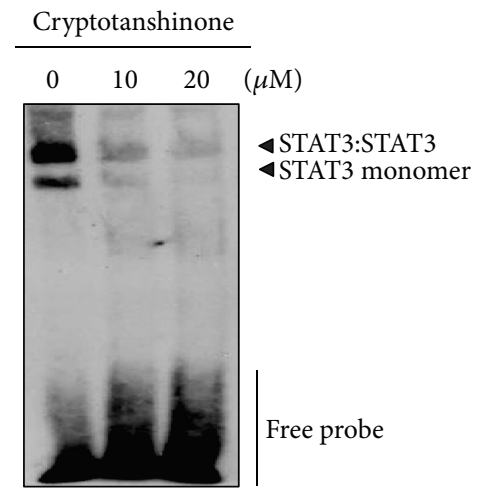

(b)

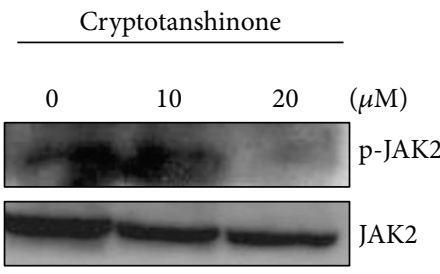

(c)

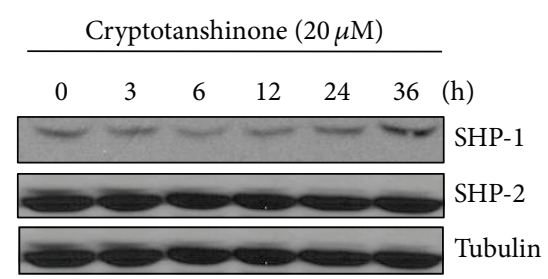

(d)

FIGURE 3: Cryptotanshinone inactivates STAT3, but not STAT5, in K562 cells. (a) Cells were treated with cryptotanshinone (0, 10, or 20 $\mu$ M) for $24 \mathrm{~h}$ (left) or $20 \mu \mathrm{M}$ for $0,6,12$, or $24 \mathrm{~h}$ (right). Cell lysates were prepared and subjected to Western blotting for phospho-STAT3 and phospho-STAT5. (b) Cells were treated with cryptotanshinone $(0,10$, or $20 \mu \mathrm{M})$ for $24 \mathrm{~h}$. Gel shift mobility assay was performed to determine the STAT3/DNA binding activity. (c) Cells were treated with cryptotanshinone $(0,10$, or $20 \mu \mathrm{M})$ for $24 \mathrm{~h}$. Western blotting was performed to detect phosphorylation of JAK2. (d) Cells were treated with $20 \mu \mathrm{M}$ cryptotanshinone for $0,3,6,12,24$, or $36 \mathrm{~h}$. Western blotting was conducted to determine the expression of SHP-1 and SHP-2.

in Figure 5(a), both tanshinone IIA and cryptotanshinone reduced phosphorylation of bcr-abl in a dose-dependent manner. Then, to test the synergy between tanshinone IIA or cryptotanshinone and imatinib, a competitive tyrosine kinase inhibitor used in the treatment of CML [30], K562 cells were cotreated with tanshinone IIA or cryptotanshinone $(0,2.5$, or $5 \mu \mathrm{M})$ in the absence or presence of imatinib $(0.25 \mu \mathrm{M})$ for $24 \mathrm{~h}$. The cell viability was significantly decreased in combination of tanshinone IIA or cryptotanshinone with imatinib in a dose-dependent manner compared to untreated control (Figure 5(b)). Tanshinone IIA remarkably showed the synergistic effect on the imatinib-induced apoptosis with CI value $=0.315$ and 0.628 at 2.5 and $5 \mu \mathrm{M}$, respectively (Figure $5(\mathrm{c})$ ). In contrast, cryptotanshinone treatment with imatinib had the synergistic effect only at $2.5 \mu \mathrm{M}(\mathrm{CI}=0.776)$ while showing the additional effect at $5 \mu \mathrm{M}(\mathrm{CI}=1.048)$ (Figure $5(c)$ ). Furthermore, combination treatment of imatinib and tanshinone IIA synergistically increased the apoptotic population of Annexin V-PI double positive stained cells to $16 \%$, while single treatment of imatinib or tanshinone IIA induced $4.96 \%$ and $9.18 \%$ apoptosis in K562, respectively (Figure $5(d))$.

\section{Discussion}

Phytochemicals are natural compounds in plants such as fruits, vegetables, beans, grains, and others. In the American Cancer Society (ACS) report in 2008, some phytochemicals may account for the beneficial effects in humans to prevent and treat many health conditions. For this reason, these 

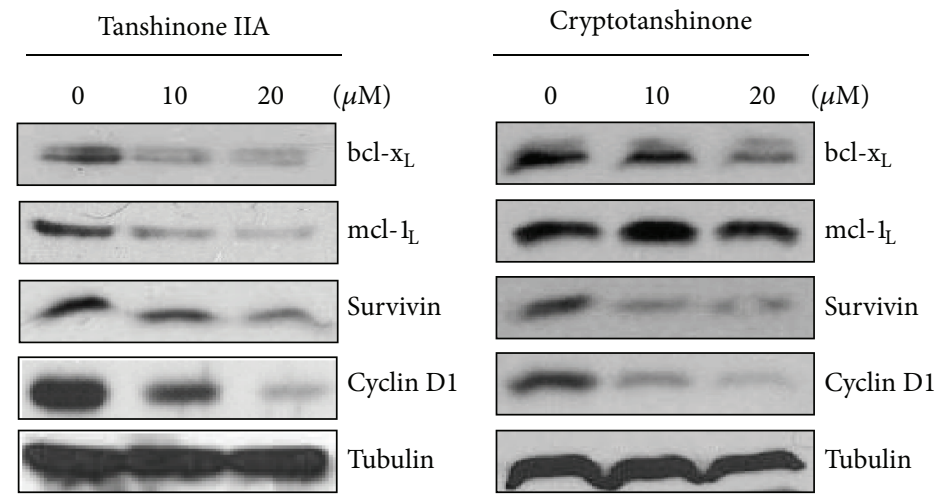

(a)

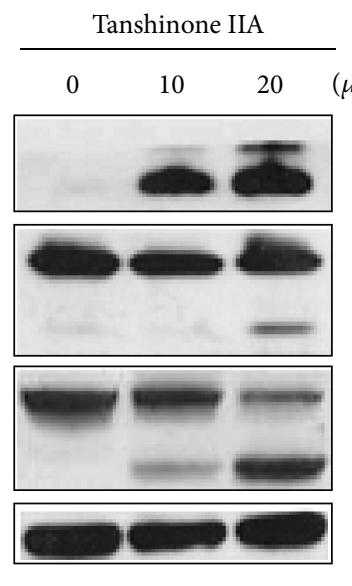

Control
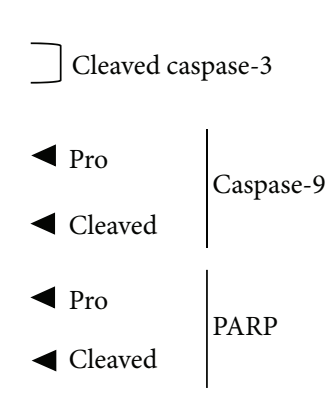

Tubulin

\section{$\frac{\text { Cryptotanshinone }}{0} \quad 10 \quad 20 \quad(\mu \mathrm{M})$}
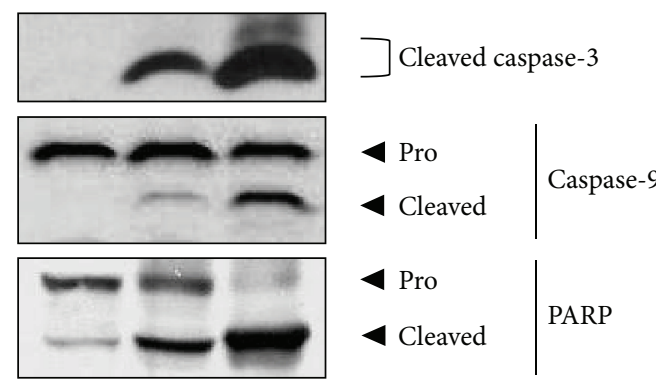

Tubulin

(b)
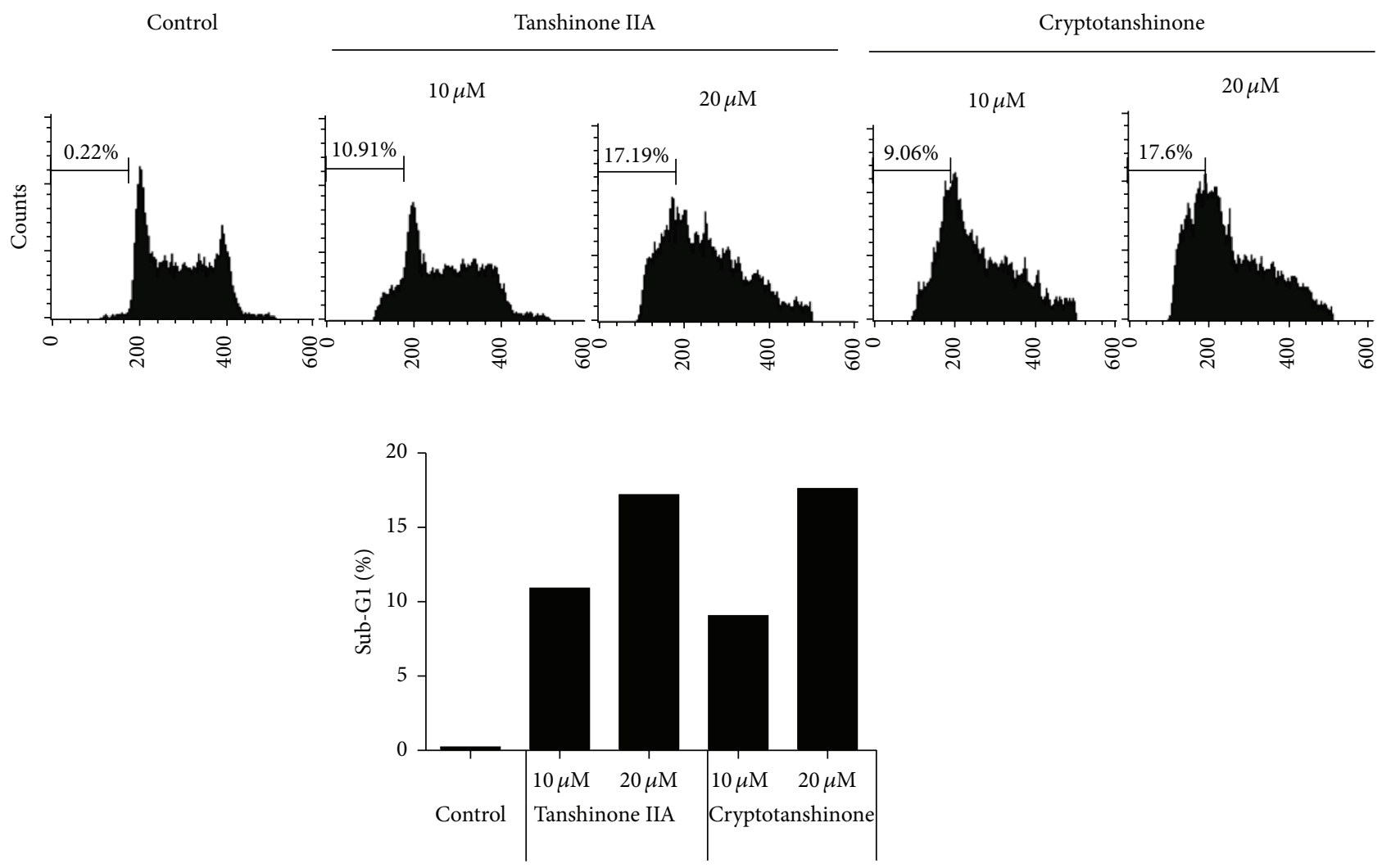

(c)

FIGURE 4: Continued. 


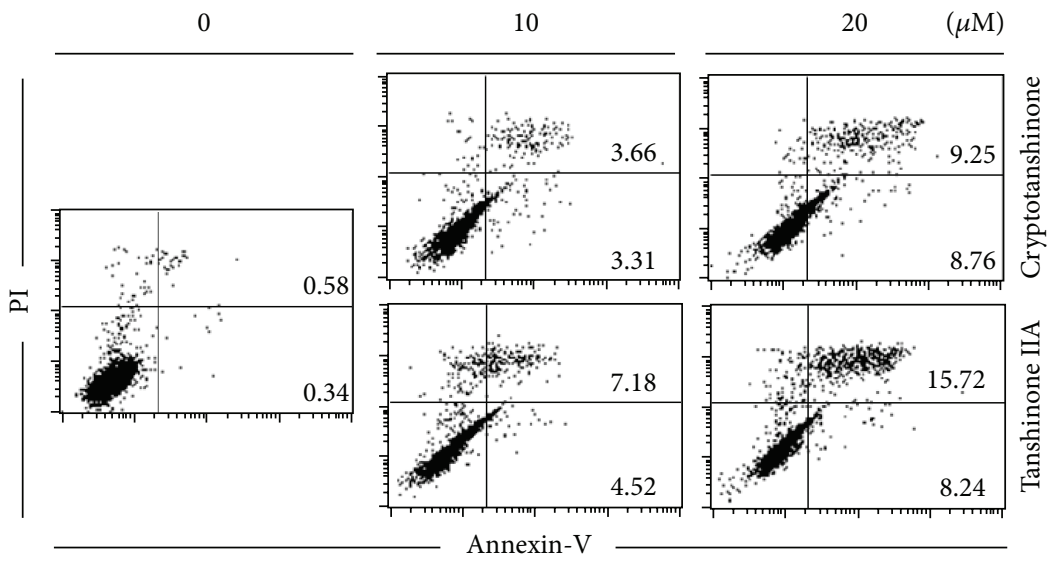

(d)

Figure 4: Tanshinone IIA and cryptotanshinone induce apoptosis in K562 cells. (a and b) Cells were treated with tanshinone IIA (left) or cryptotanshinone (right) for $24 \mathrm{~h}$. Cell lysates were prepared and subjected to Western blotting to detect expression of apoptosis-related proteins bcl- $\mathrm{x}_{\mathrm{L}}, \mathrm{mcl}_{\mathrm{L}}$, surviving, and cyclin D1 (a) and caspase-3, caspase-9, and PARP (b). (c) Flow cytometry analysis of sub-G1 apoptotic DNA fraction of the cells treated with tanshinone IIA or cryptotanshinone $(0,10$, or $20 \mu \mathrm{M})$ for $24 \mathrm{~h}$. After fixation in $75 \%$ ethanol, cells were stained with PI and analyzed by flow cytometry. (d) Cells were treated with tanshinone IIA or cryptotanshinone $(10$ or $20 \mu \mathrm{M})$ for $24 \mathrm{~h}$. Percentage of apoptotic cells in tanshinone IIA or cryptotanshinone-treated cells by Annexin V-PI staining. Each experiment was repeated three times.

phytochemicals have been thought as valuable materials to develop new therapeutic drug or dietary supplement. Recently, numerous papers have reported the potential of phytochemicals to ameliorate the various diseases such as cancer, inflammation, metabolic syndrome, and cardiovascular disease. In the present study, we comparatively investigated the anticancer mechanism of tanshinone IIA and cryptotanshinone from S. miltiorrhiza in CML, a form of leukemia characterized by the increased and unregulated growth of predominantly myeloid cells in the bone marrow [31]. Our group recently reported that tanshinone IIA induces apoptosis through activation of c-jun $\mathrm{N}$-terminal kinase in KBM-5 cells [18]. Ge et al. reported that cryptotanshinone mediates cell cycle arrest and apoptosis of multidrug-resistant K562/ADM cells by inactivating eukaryotic initiation factor $4 \mathrm{E}$ [10]. Additionally, we also reported that cryptotanshinone enhances TNF- $\alpha$-induced apoptosis in KBM-5 cells [11]. Nonetheless, the molecular mechanisms leading to anti-CML properties of tanshinone IIA and cryptotanshinone are not fully understood yet.

STAT is one of the important transcriptional factor families and plays crucial roles as a molecular target for cancer prevention and therapy [32]. STAT family consists of 7 different subfamilies STAT1, 2, 3, 4, 5a, 5b, and 6, and STAT3 and 5 are constitutively activated in cancer cells. STAT 3 and 5 are activated by nonreceptor tyrosine kinases of the Janus family (JAK) and c-Src [33, 34], and protein tyrosine phosphatases (PTPs) such as Src homology 2 domaincontaining phosphatases (SHPs), phosphatase and tensin homolog (PTEN), and suppressor of cytokine signaling proteins (SOCS) are also linked to STAT signaling [35]. Therefore, the JAK/STAT3 or 5 signaling has been thought as a valuable molecular target for cancer therapy $[34,36]$.
In our study, we found that both tanshinone IIA and cryptotanshinone reduced the phosphorylation of JAK2, an upstream kinase of STATs, in K562 CML cells. However, the effects of tanshinone IIA and cryptotanshinone on STAT activation were clearly different in K562 cells. Tanshinone IIA reduced the phosphorylation of STAT5, but not STAT3, and consistently prevented the STAT5/DNA binding in the cells. In contrast, cryptotanshinone inactivated STAT3, but not STAT5, at posttranslational and transcriptional levels. In addition, tanshinone IIA induced the expression of SHP-1 and -2 whereas cryptotanshinone increased the expression of SHP-1, but not SHP-2, in K562 cells (Figure 6).

The JAK/STAT signaling is involved in oncogenesis and cancer progression through upregulation of antiapoptotic genes [37]. Tanshinone IIA and cryptotanshinone commonly repressed the expression of bcl- $\mathrm{x}_{\mathrm{L}}$, survivin and cyclin D1 in $\mathrm{K} 562$ cells. In contrast, only tanshinone IIA, but not cryptotanshinone, decreased the $\mathrm{mcl}-\mathrm{l}_{\mathrm{L}}$ expression. Apoptosis induction by tanshinone IIA or cryptotanshinone was confirmed by activation of caspase- 9 and -3 , cell cycle analysis and nuclear staining using PI (Figure 6). Although tanshinone IIA and cryptotanshinone exerted anti-CML activities in a different way by targeting the distinct STAT signaling, there was no significant difference in the induction of apoptosis by them. Further studies are necessary such as gene silencing for SHP-2 or $m c l-1_{L}$ to verify the precise mechanisms responsible for the different regulation between tanshinone IIA and cryptotanshinone against CML cells in the near future. Using stable cells overexpressed STAT3 or 5 will be also beneficial tools to prove the anti-CML mechanisms.

Bcr-Abl selective tyrosine kinase inhibitor, imatinib (marketed by Novartis as Gleevec), has been extensively used for CML therapy [30]. However, despite of its specific therapeutic 


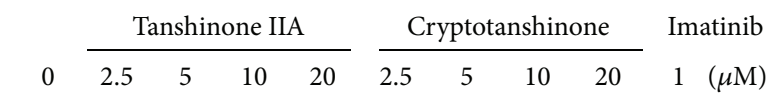

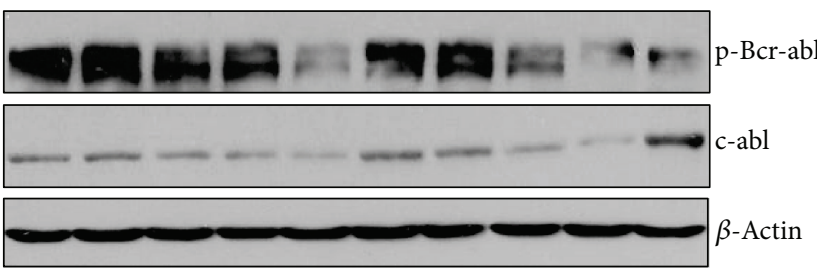

(a)
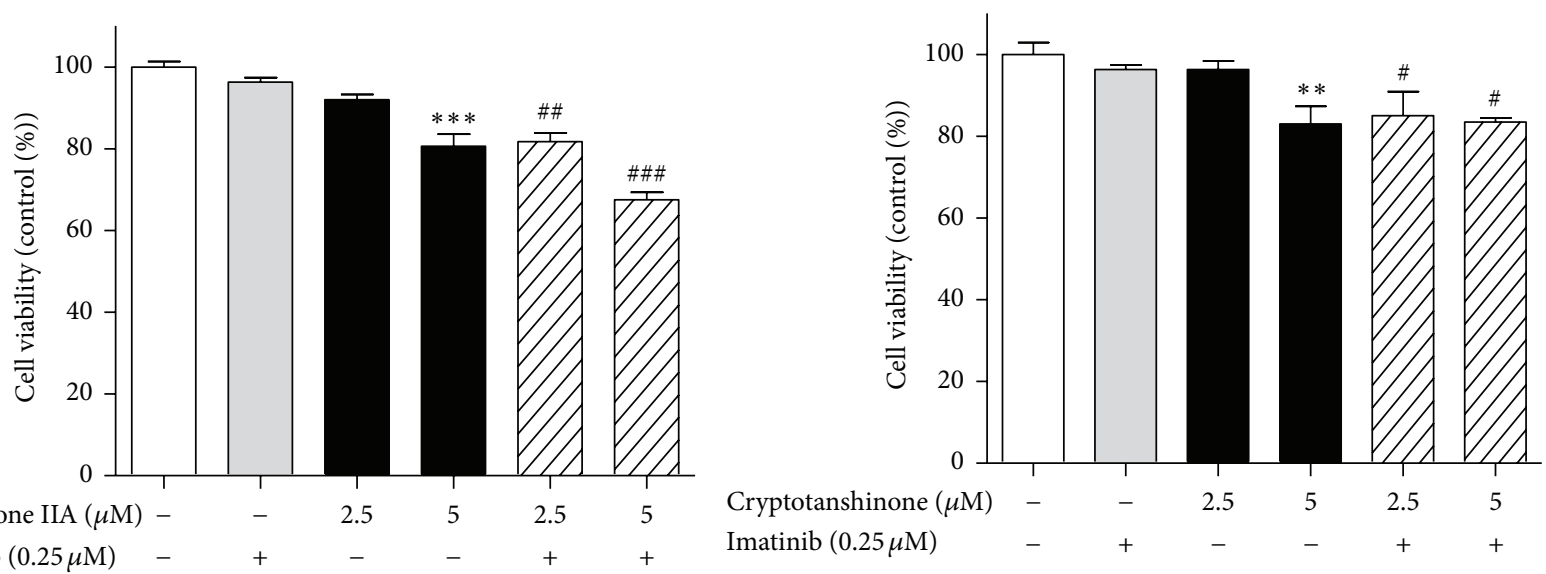

(b)

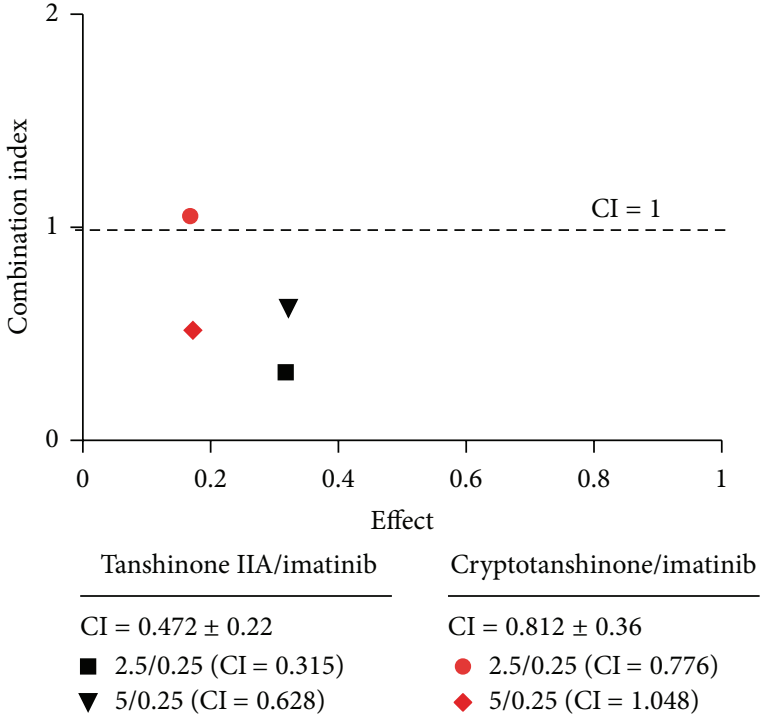

(c)
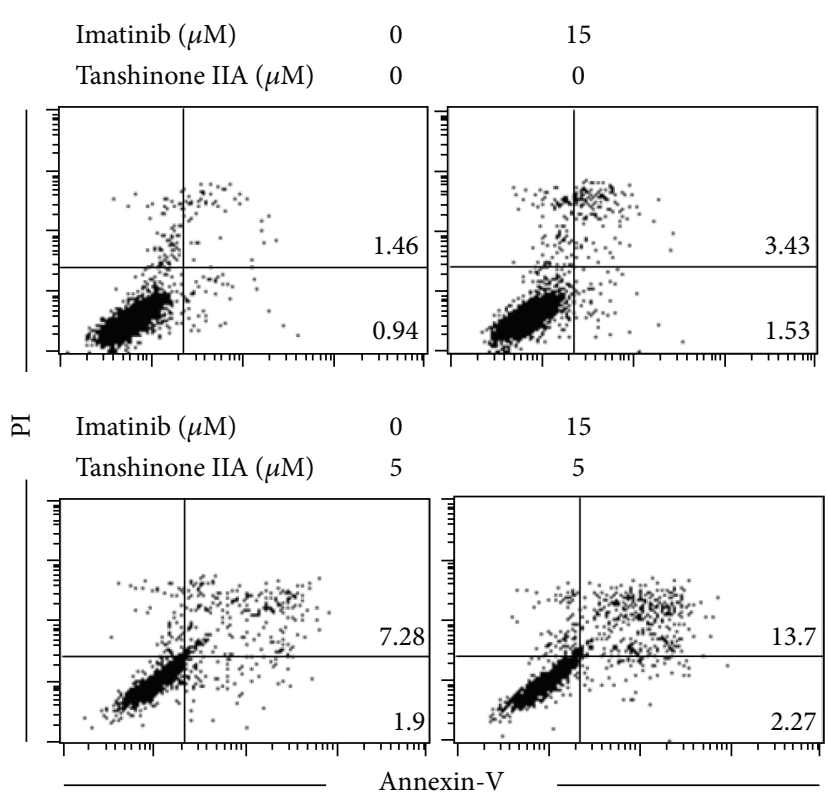

(d)

FIGURE 5: Tanshinone IIA and cryptotanshinone with imatinib synergistically inhibit the viability of K562 cells. (a) Cells were treated with various concentrations of tanshinone IIA or cryptotanshinone $(0,2.5,5,10$, or $20 \mu \mathrm{M})$ for $24 \mathrm{~h}$. Cell lysates were prepared and subjected to Western blotting for phospho-bcr-abl. (b) Cells were treated with tanshinone IIA (left) or cryptotanshinone (right) and/or imatinib for $24 \mathrm{~h}$. Cell viability was measured by MTT assay. (c) The combination index (CI) between two drugs was determined by Chou-Talalay method and CalcuSyn software (Biosoft, Ferguson, MO, USA). (d) Cells were treated with tanshinone IIA and imatinib for $24 \mathrm{~h}$ at the same time. Percentage of apoptotic cells in cotreated cells by Annexin V-PI double staining. 


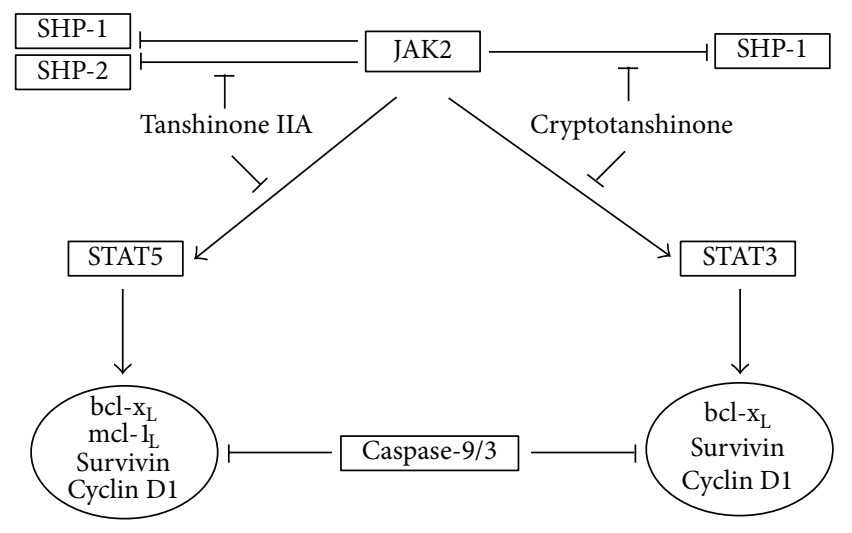

FIGURE 6: Schematic diagram indicating the effect of tanshinone IIA and cryptotanshinone on JAK/STAT signaling and apoptosis pathways in CML cells.

effect for CML, serious adverse effects and cost problem can limit the use of imatinib. In the current study, we tested the possibility that tanshinone IIA or cryptotanshinone can stimulate anti-CML effect induced by imatinib by lowering dosage in K562 cells. Our data revealed that tanshinone IIA enhanced imatinib-induced cell death more effectively than cryptotanshinone, with CI value $<1$ even at $2.5 \mu \mathrm{M}$, determined by Chou-Talalay method and CalcuSyn software, implying significant synergy between tanshinone IIA and imatinib as a potent combination therapy for CML. However, additional experiments are required using in vivo mouse xenograft model to validate the in vitro studies.

In summary, tanshinone IIA inhibited JAK2/STAT5 signaling, whereas cryptotanshinone targets the JAK2/STAT3 in K562 cells. Furthermore, tanshinone IIA enhanced the expression of both SHP-1 and -2, while cryptotanshinone regulated the expression of only SHP-1. Also, both tanshinone IIA and cryptotanshinone attenuated the expression of STATrelated genes such as bcl- $\mathrm{x}_{\mathrm{L}}$, survivin, and cyclin D1.

\section{Conclusion}

Our findings clearly demonstrate that anticancer activity of tanshinone IIA and cryptotanshinone is mediated by the distinct JAK/STAT3/5 and SHP1/2 signaling in K562 cells. Of note, tanshinone IIA showed more potential for the synergy with imatinib compared with cryptotanshinone as a potent candidate for combination therapy.

\section{Authors' Contribution}

Ji Hoon Jung, Tae-Rin Kwon, and Soo-Jin Jeong contributed equally to this work.

\section{Acknowledgment}

This work was supported by the Korea Science and Engineering Foundation (KOSEF) Grant funded by the Korea government (MEST) (no. 2011-0063466).

\section{References}

[1] L. Zhou, Z. Zuo, and M. S. S. Chow, "Danshen: an overview of its chemistry, pharmacology, pharmacokinetics, and clinical use," Journal of Clinical Pharmacology, vol. 45, no. 12, pp. 1345-1359, 2005.

[2] A.-H. Liu, L. Li, M. Xu, Y.-H. Lin, H.-Z. Guo, and D.-A. Guo, "Simultaneous quantification of six major phenolic acids in the roots of Salvia miltiorrhiza and four related traditional Chinese medicinal preparations by HPLC-DAD method," Journal of Pharmaceutical and Biomedical Analysis, vol. 41, no. 1, pp. 48$56,2006$.

[3] L. Ma, X. Zhang, H. Guo, and Y. Gan, "Determination of four water-soluble compounds in Salvia miltiorrhiza Bunge by high-performance liquid chromatography with a coulometric electrode array system," Journal of Chromatography B, vol. 833, no. 2, pp. 260-263, 2006.

[4] X. Wang, S. L. Morris-Natschke, and K.-H. Lee, "New developments in the chemistry and biology of the bioactive constituents of Tanshen," Medicinal Research Reviews, vol. 27, no. 1, pp. 133148, 2007.

[5] H.-C. Bi, Z. Zuo, X. Chen et al., "Preclinical factors affecting the pharmacokinetic behaviour of tanshinone IIA, an investigational new drug isolated from Salvia miltiorrhiza for the treatment of ischaemic heart diseases," Xenobiotica, vol. 38, no. 2, pp. 185-222, 2008.

[6] M.-J. Don, C.-C. Shen, W.-J. Syu, Y.-H. Ding, and C.-M. Sun, "Cytotoxic and aromatic constituents from Salvia miltiorrhiza," Phytochemistry, vol. 67, no. 5, pp. 497-503, 2005.

[7] M. Gu, G. Zhang, Z. Su, and F. Ouyang, "Identification of major active constituents in the fingerprint of Salvia miltiorrhiza Bunge developed by high-speed counter-current chromatography," Journal of Chromatography A, vol. 1041, no. 1-2, pp. 239243, 2004.

[8] W. Chen, L. Liu, Y. Luo et al., "Cryptotanshinone activates p38/ JNK and inhibits Erk1/2 leading to caspase-independent cell death in tumor cells," Cancer Prevention Research, vol. 5, pp. 778-787, 2012.

[9] W. Chen, Y. Lu, G. Chen, and S. Huang, "Molecular evidence of cryptotanshinone for treatment and prevention of human cancer," Anti-cancer Agents in Medicinal Chemistry. In press.

[10] Y. Ge, R. Cheng, Y. Zhou et al., "Cryptotanshinone induces cell cycle arrest and apoptosis of multidrug resistant human chronic myeloid leukemia cells by inhibiting the activity of eukaryotic initiation factor 4E," Molecular and Cellular Biochemistry, vol. 368, pp. 17-25, 2012.

[11] J.-H. Kim, S.-J. Jeong, T.-R. Kwon et al., "Cryptotanshinone enhances TNF- $\alpha$-induced apoptosis in chronic myeloid leukemia KBM-5 cells," Apoptosis, vol. 16, no. 7, pp. 696-707, 2011.

[12] H. J. Lee, D. B. Jung, E. J. Sohn et al., "Inhibition of hypoxia inducible factor alpha and astrocyte-elevated gene-1 mediates cryptotanshinone exerted antitumor activity in hypoxic PC-3 cells," Evidence-Based Complementary and Alternative Medicine, vol. 2012, Article ID 390957, 13 pages, 2012.

[13] C.-Y. Cheng and C.-C. Su, "Tanshinone IIA may inhibit the growth of small cell lung cancer H146 cells by up-regulating the $\mathrm{Bax} / \mathrm{Bcl}-2$ ratio and decreasing mitochondrial membrane potential," Molecular Medicine Reports, vol. 3, no. 4, pp. 645650, 2010.

[14] C.-C. Su, "Tanshinone IIA potentiates the efficacy of 5-FU in Colo205 colon cancer cells in vivo through downregulation of 
P-gp and LC3-II," Experimental and Therapeutic Medicine, vol. 3, no. 3, pp. 555-559, 2012.

[15] S.-H. Won, H.-J. Lee, S.-J. Jeong et al., “Tanshinone IIa induces mitochondria dependent apoptosis in prostate cancer cells in association with an inhibition of phosphoinositide 3-kinase/ AKT pathway," Biological and Pharmaceutical Bulletin, vol. 33, no. 11, pp. 1828-1834, 2010.

[16] S.-H. Won, H.-J. Lee, S.-J. Jeong, J. Lü, and S.-H. Kim, "Activation of p53 signaling and inhibition of androgen receptor mediate tanshinone IIA induced G1 arrest in LNCaP prostate cancer cells," Phytotherapy Research, vol. 26, no. 5, pp. 669-674, 2012.

[17] S. Xu and P. Liu, "Tanshinone II-A: new perspectives for old remedies," Expert Opinion on Therapeutic Patents, vol. 23, pp. 149-153, 2013.

[18] S. M. Yun, S. J. Jeong, J. H. Kim et al., "Activation of C-Jun Nterminal kinase mediates tanshinone IIA-induced apoptosis in KBM-5 chronic myeloid leukemia cells," Biological \& Pharmaceutical Bulletin, vol. 36, pp. 208-214, 2013.

[19] R. Buettner, L. B. Mora, and R. Jove, "Activated STAT signaling in human tumors provides novel molecular targets for therapeutic intervention," Clinical Cancer Research, vol. 8, no. 4, pp. 945-954, 2002.

[20] J. Vera, K. Rateitschak, F. Lange, C. Kossow, O. Wolkenhauer, and R. Jaster, "Systems biology of JAK-STAT signalling in human malignancies," Progress in Biophysics and Molecular Biology, vol. 106, no. 2, pp. 426-434, 2011.

[21] C. Tang, H.-L. Xue, H.-B. Huang, and X.-G. Wang, “Tanshinone IIA inhibits constitutive STAT3 activation, suppresses proliferation, and induces apoptosis in rat C6 glioma cells," Neuroscience Letters, vol. 470, no. 2, pp. 126-129, 2010.

[22] D.-S. Shin, H.-N. Kim, K. D. Shin et al., "Cryptotanshinone inhibits constitutive signal transducer and activator of transcription 3 function through blocking the dimerization in DU145 prostate cancer cells," Cancer Research, vol. 69, no. 1, pp. 193-202, 2009.

[23] H.-S. Choi, D.-I. Cho, H.-K. Choi, S. Y. Im, S.-Y. Ryu, and K.-M. Kim, "Molecular mechanisms of inhibitory activities of tanshinones on lipopolysaccharide-induced nitric oxide generation in RAW 264.7 cells," Archives of Pharmacal Research, vol. 27, no. 12, pp. 1233-1237, 2004.

[24] J. M. Hur, J. S. Shim, H. J. Jung, and H. J. Kwon, "Cryptotanshinone but not tanshinone IIA inhibits angiogenesis in vitro," Experimental and Molecular Medicine, vol. 37, no. 2, pp. 133-137, 2005.

[25] T.-C. Chou, "Drug combination studies and their synergy quantification using the chou-talalay method," Cancer Research, vol. 70, no. 2, pp. 440-446, 2010.

[26] W. X. Li, "Canonical and non-canonical JAK-STAT signaling," Trends in Cell Biology, vol. 18, no. 11, pp. 545-551, 2008.

[27] P. Sansone and J. Bromberg, "Targeting the interleukin-6/Jak/ stat pathway in human malignancies," Journal of Clinical Oncology, vol. 30, no. 9, pp. 1005-1014, 2012.

[28] H. Duan, K. Orth, A. M. Chinnaiyan et al., "ICE-LAP6, a novel member of the ICE/Ced-3 gene family, is activated by the cytotoxic T cell protease granzyme B," Journal of Biological Chemistry, vol. 271, no. 28, pp. 16720-16724, 1996.

[29] C. L. Sawyers, C. T. Denny, and O. N. Witte, "Leukemia and the disruption of normal hematopoiesis," Cell, vol. 64, no. 2, pp. 337-350, 1991.
[30] B. J. Druker, S. Tamura, E. Buchdunger et al., "Effects of a selective inhibitor of the Abl tyrosine kinase on the growth of BcrAbl positive cells," Nature Medicine, vol. 2, no. 5, pp. 561-566, 1996.

[31] S. Faderl, M. Talpaz, Z. Estrov, and H. M. Kantarjian, "Chronic myelogenous leukemia: biology and therapy," Annals of Internal Medicine, vol. 131, no. 3, pp. 207-219, 1999.

[32] B. B. Aggarwal, G. Sethi, K. S. Ahn et al., "Targeting signaltransducer-and-activator-of-transcription-3 for prevention and therapy of cancer: Modern target but ancient solution," Annals of the New York Academy of Sciences, vol. 1091, pp. 151-169, 2006.

[33] C. M. Silva, "Role of STATs as downstream signal transducers in Src family kinase-mediated tumorigenesis," Oncogene, vol. 23, no. 48, pp. 8017-8023, 2004.

[34] O. V. Smirnova, T. Y. Ostroukhova, and R. L. Bogorad, "JAKSTAT pathway in carcinogenesis: is it relevant to cholangiocarcinoma progression?" World Journal of Gastroenterology, vol. 13, no. 48, pp. 6478-6491, 2007.

[35] Y. Han, H. M. Amin, B. Franko, C. Frantz, X. Shi, and R. Lai, "Loss of SHP1 enhances JAK3/STAT3 signaling and decreases proteosome degradation of JAK3 and NPM-ALK in ALK+ anaplastic large-cell lymphoma," Blood, vol. 108, no. 8, pp. 27962803, 2006.

[36] T. Hirano, K. Ishihara, and M. Hibi, "Roles of STAT3 in mediating the cell growth, differentiation and survival signals relayed through the IL-6 family of cytokine receptors," Oncogene, vol. 19, no. 21, pp. 2548-2556, 2000.

[37] J. Turkson, "STAT proteins as novel targets for cancer drug discovery," Expert Opinion on Therapeutic Targets, vol. 8, no. 5, pp. 409-422, 2004. 


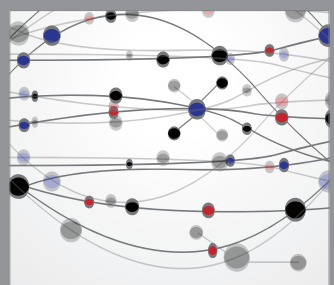

The Scientific World Journal
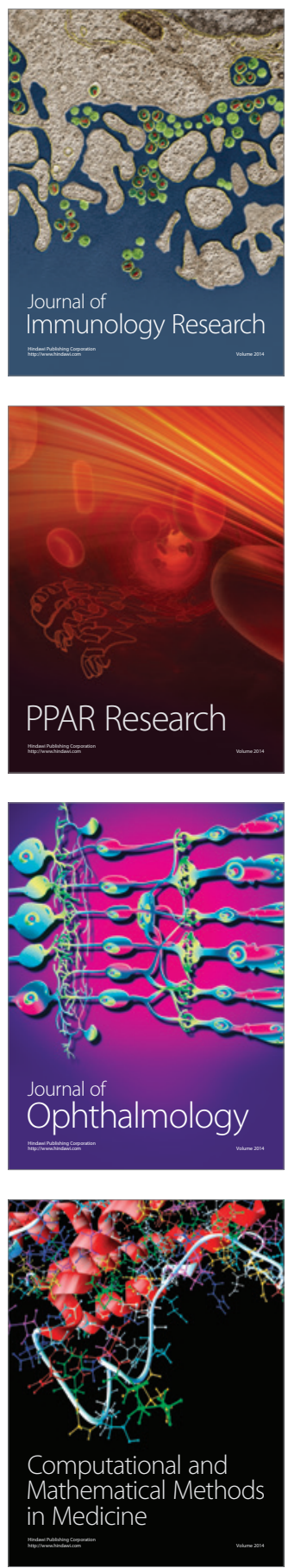

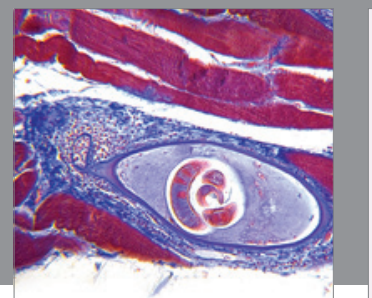

Gastroenterology

Research and Practice
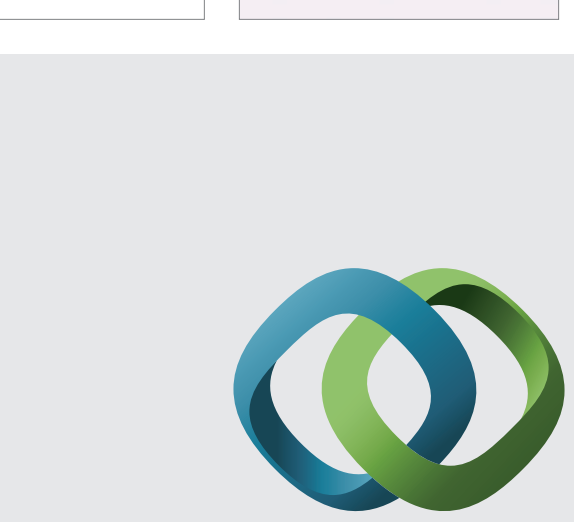

\section{Hindawi}

Submit your manuscripts at

http://www.hindawi.com
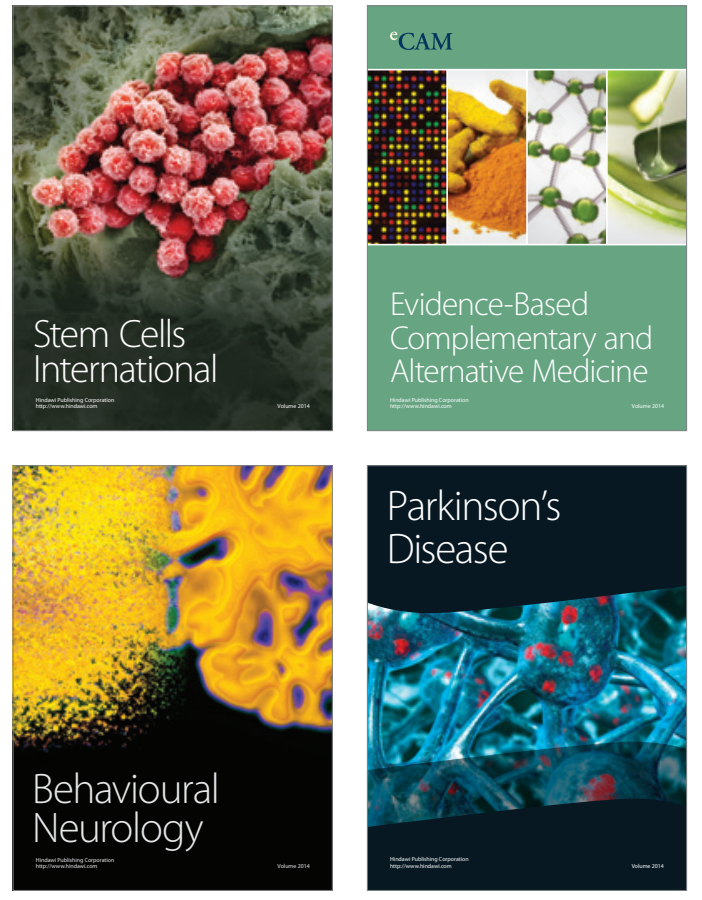
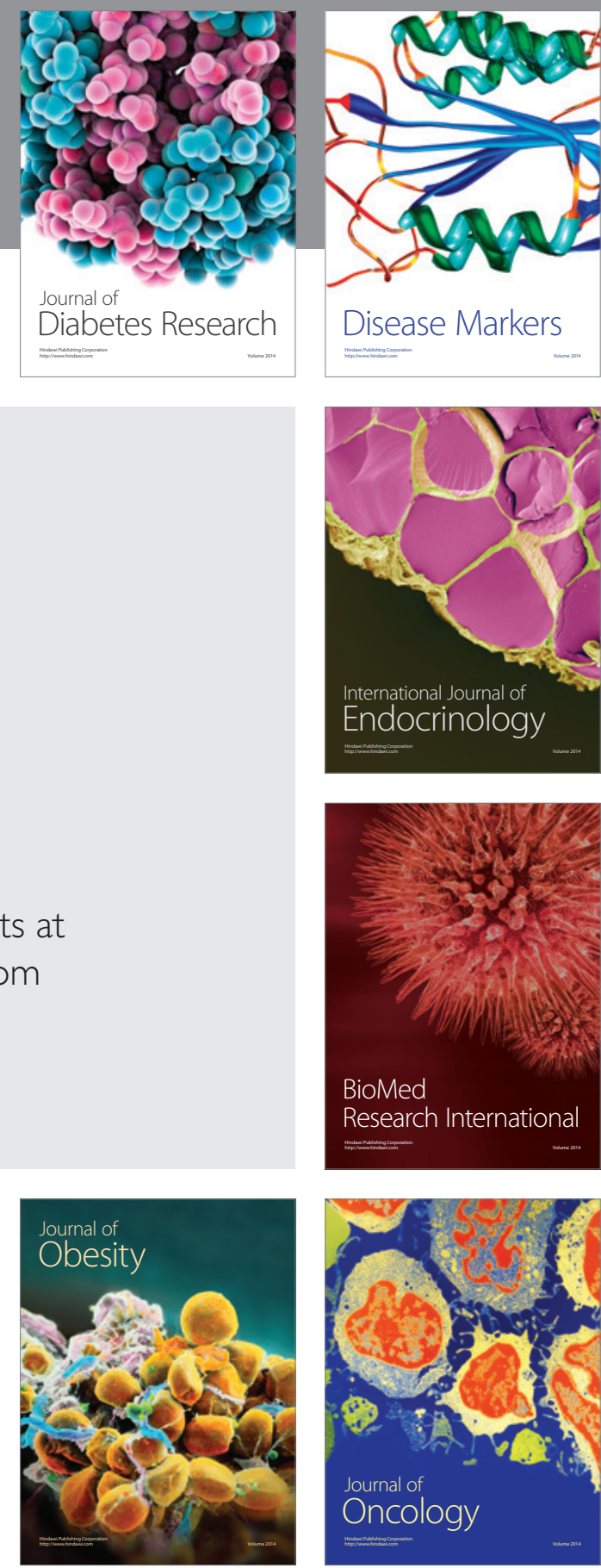

Disease Markers
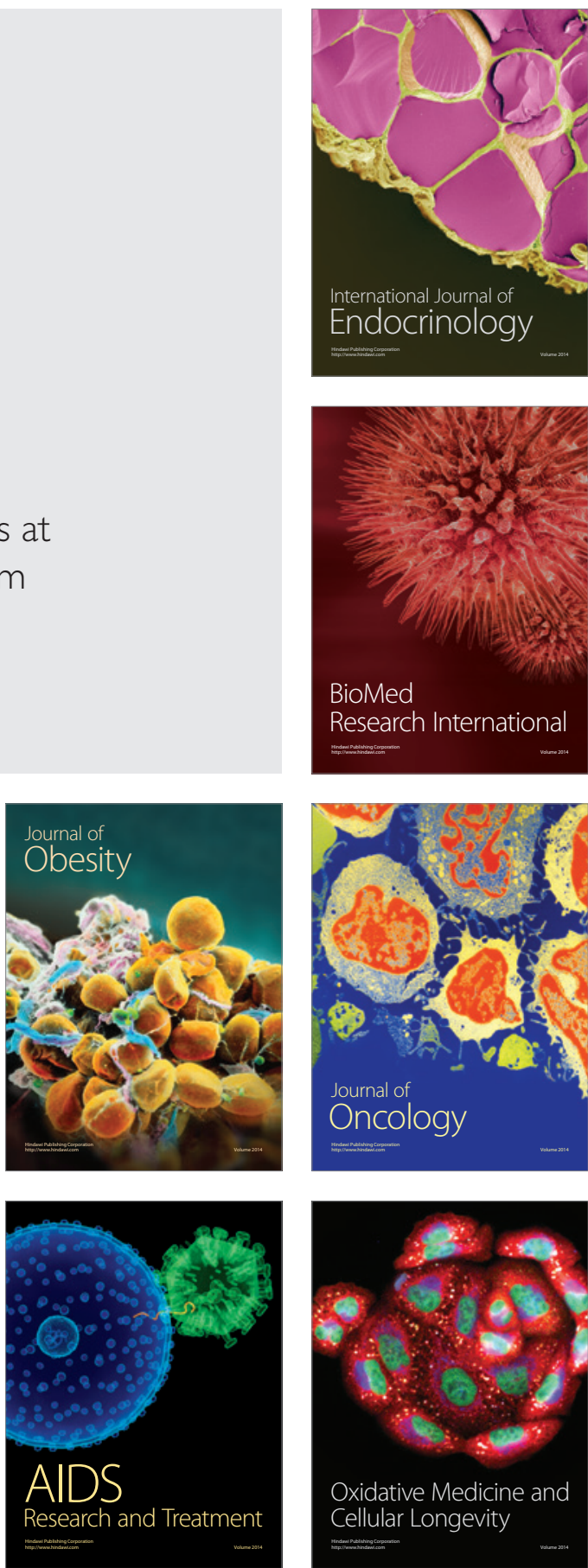